\title{
Development of Novel Classifying System to Identify the Right Sense of Audio Conversation in Social Networks using Deep Convolution Neural Network
}

\author{
P.Nirupama, E. Madhusudhana Reddy
}

\begin{abstract}
Social media has paved a new way for communication and interacting with others. The use of social media differs according to the socio-cultural, demographic and psychological aspects of individuals. People chat, share ideas and visual material, and feel that they satisfy their needs of belonging along with the groups they have joined. Social networks is not only a area of freedom where persons express themselves openly or furtively, but also an area where several ways of violence emerge or even a means used for some aspects of violence.. The present research throws light on a few of the regular and trendy methods of abuse and risks faced by the users of social media. Develop a system to identify abusing audio file by an individual on a people/ group based on common language, race, sexual preferences, religion, or nationality. We examine a new model from machine learning, namely deep machine learning by probing design configurations of deep Convolutional Neural Networks (CNN) and the impact of different hyper-parameter settings in identifying the negative aspects in social media. Deep CNN automatically generate powerful features by hierarchical learning strategies from massive amounts of training data with a minimum of human interaction or expert process knowledge. An application of the proposed method demonstrates excellent results with low false alarm rates for Twitter data.
\end{abstract}

\section{INTRODUCTION}

We live in a world where the whole thing is an illustration or an illusion. The matrix is real and always with us. Then, who cares what is right or wrong? Despite the use of easiness it provides to share different views and emotions, we can hardly say that social networks has noticeably changed and shaped the real world. Twitter, Facebook and the other social networks function as platforms where individuals express their opinions about anything, anytime. Nevertheless, certainty and continuity of these ideas are quite debatable. Instantaneous approvals and quick transformations with regard to different ideas make us question the reliability of this medium.

Recently, the new trend called internet banging, has been reported by media, in which persons caught up in gangs or neighborhood factions use social media sites such as Twitter and Facebook to make threats of violence which may result in slaughter or ill-treatment. These examples clearly indicate the extremities of the Internet abuses.

Revised Manuscript Received on July 22, 2019.

P.Nirupama, Research Scholar, Computer Science, Bharathiar University, Coimbatore, Tamil Nadu

E. Madhusudhana Reddy, Professor, Computer Science and Engineering, Guru Nanak Institutions Technical Campus, India

E.Mail: e_mreddy@yahoo.com, nirupama.cse1@gmail.com
Cyber-bullying can be defined as harassment which is exercised by using electronic technology including "mobiles, computers, and tabs as well as communication tools including social media, audio file, chat, and websites intractable and recurring damage inflict through the use of electronic devices".

Social media gives every person the chance to carve their own scenarios and turn into heroes or heroines. The way these people use social networks would naturally differ as these scenarios are written by those having different values. Probably social networks makes everything, either positive or negative, is the world more conspicuous or normal. A social media produce the associations surrounded by a native structure of meaning and discourse. The main purpose of joining the social networks and surviving in that territory is the need of individuals to express themselves to the others and also expect to be perceived important by them.

Overall, restrictions on freedom inevitably lead to breakup of social values. The members of a society that feels continuous oppression is somehow obliged to express themselves by resorting to violence. By way of social media that provide the placate of getting away from facts, the practice of violence becomes rather easier. Since social networks creates the dilemma of being somewhere and also not really being there, individuals may normalize violence by continually being exposed to loss of values in this hazy reality As violence heightens, more restrictions on freedoms are imposed, which in turn brings about a vicious circle.

\section{LITERATURE REVIEW}

As per Althusser and the others (Üşür,1997), the debate over ideology aim to put forth how. In fact, Social networks create the stuffing of violence because needless to say, ideologies arise from needs. In this logic, social networks produce its own philosophy in accord with the needs. Florea(2013) said that violent behavior has so far been an vital part of our life and assert that either as children or grownups, we may be the "aggressors, victims or witnesses of an aggression"(p. 350). In reality as a result of political, economic or social reasons, in today's era any event may simply trigger deep-rooted aggression. Hinduja \& Patchin as cited in Manktelowa, \& Taylor, 2014 states that, cyber-bullying can be defined as a form bullying which is exercised by using " mobiles, computers, 
and tabs as well as communication tools in social media sites, audio file, chat, and sites headstrong and recurring mischief through the use of electronic tools".

VanLaer(2013) defines the potentially harmful effects of cyber harassment as "emotional distress... withdrawal from social media even life itself". Ybarra and Mitchell(2004) indicate that cyber bullying may be in different ways such as sending unwanted derogatory or threatening comments, rumors, pictures or videos that are offensive or embarrassing by audio file, email, chat, or posting on websites including social networking sites (Görzig \& Frumkin, 2013). As per R.Sennet (2006/2009), the main purpose of joining the social networks and surviving in that territory is the need of individuals to express themselves to the others and also expect to be perceived important by them.

Masuda 2010, Social media is an important area to realize social practices and sharing of realities. The way of using the information shared on social media brings up significant effects in economic, social and cultural dimensions. Habermas (as cited in Slattery, 2003/2007, p.437), public sphere is an area where individuals think and ponder about common topics, participate insensible discussions and come to conclusion on particular issues through particular processes, means and situations. According to Güngör (2011) into day's world surrounded with computer technology, the issue that can be associated with ethics is simply privacy. In other words, privacy managed to remain unchanged in the world where capital is made everything a commodity until the threatening advent and development of audio file-visual techniques.

\section{AIM AND OBJECTIVES}

The aim of this thesis is to develop novel Deep Convolution Neural Networks system to perfectly identify and classify negative aspects in social networks when an individual or a people/ group share/post an abusing audio file on an individual or a people/ group based on common language, race, sexual preferences, religion, or nationality, and notifying them that they are doing wrong as per law of any country with respect to Social Networks. By ensuring these services, we aim at helping an individual or a people/ group to overcome the distrust in engaging through Social Networks with others over the Internet and increasing their confidence in Novel Classifying System Using Deep Convolution Neural Network. In order to achieve this aim, the objectives of this thesis are as follows.

$>\quad$ Developed a system to extract audio file from Twitter to know the Purpose behind the usage of social networking sites.

$>\quad$ Designed a system to identify the right sense of conversation of Audio file in social networks based on common language, race, sexual preferences, religion, or nationality using Deep Convolution Neural Network.

$>$ Designed and developed a fair system to monitor $24 \mathrm{X}$ 7 and identify the right sense of conversation of audio file in social networks using Deep Convolution Neural Network. $>\quad$ Proposed an efficient Novel Classifying System.

\section{METHODOLOGY}

The proposed method in this thesis is divided into four main parts: (1) data acquisition, (2) data preprocessing, (3) features extraction and fusion, and (4) pattern classification algorithm. First, collect/ extract audio file from Twitter accounts of some of our friends to know the purpose behind the usage of social networking sites and preprocess the audio file. Then, the audio file is extracted by Deep Convolution Neural Network, and these features are merged with the audio file feature. Finally, the pattern recognition Softmax tool is used for constitution recognition as positive or negative.

\section{DESIGN AND DEVELOPMENT OF A NOVEL CLASSIFYING SYSTEM}

The goal of novel classifying system design is a set of test case inputs that provide consistent coverage across the test space at a known depth. This results a set of test cases that focus on driving the functionality independent of the implementation. A deep CNN with softmax model creation can divide as three steps.

1. Define the scope of the model.

2. Identify the attributes audio file and enumerate the values.

3. Apply data using deep CNN with softmax tool and classify the data as normal or abuse.

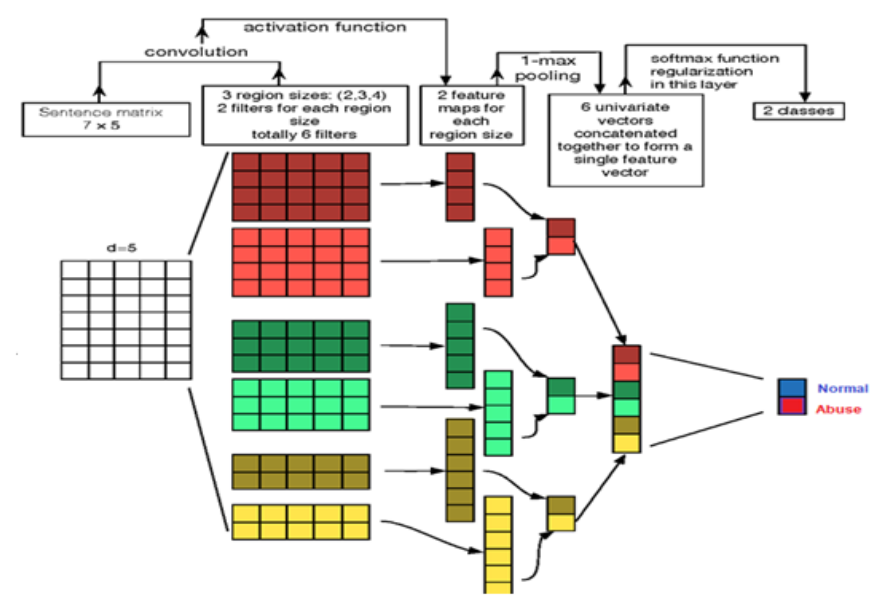

Figure 1: Illustration of a Deep Convolution Neural Network (CNN) architecture for audio file converted to block of sentences classification

In Figure 1, we depict three filter region sizes: 2, 3 and 4, each of which has 2 filters. Every filter performs convolution on the sentence matrix and generates (variable-length) feature maps. Then 1-max pooling is performed over each map, i.e., the largest number from each feature map is recorded. Thus a univariate feature vector is generated from all six maps, and these 6 features are concatenated to form a feature vector for the penultimate layer. The final soft-max layer then receives this feature vector as input and uses it to classify the sentence; 
here we assume binary classification and hence depict two possible output states Normal and Abuse.

\section{DEEP CONVOLUTIONAL NEURAL NETWORK ALGORITHM}

Convolutional neural networks are deep artificial neural networks that are used primarily to classify images (e.g. name what they see). Convolutional networks perform optical character recognition (OCR) to digitize audio file and make natural-language processing possible on analog and hand-written documents, where the images are symbols to be transcribed. CNNs can also be applied to sound when it is represented visually as a spectrogram. More recently, convolutional networks have been applied directly to audio file analytics as well as graph data with graph convolutional networks.

Activation functions: So what does an artificial neuron do? Simply put, it calculates a "weighted sum" of its input, adds a bias and then decides whether it should be "fired" or not ( yeah right, an activation function does this, but let's go with the flow for a moment ).

So consider a neuron.

$$
Y=\sum(\text { weight } * \text { input })+\text { bias }
$$

Now, the value of $\mathrm{Y}$ can be anything ranging from -inf to +inf. The neuron really doesn't know the bounds of the value. So how do we decide whether the neuron should fire or not (why this firing pattern? Because we learnt it from biology that's the way brain works and brain is a working testimony of an awesome and intelligent system).

The first thing that comes to our minds would be ReLu function.

ReLu: First, comes the ReLu function, $\mathrm{A}(\mathrm{x})=\max (0, \mathrm{x})$. The ReLu function is as shown above. It gives an output $\mathrm{x}$ if $\mathrm{x}$ is positive and 0 otherwise. At first look this would look like having the same problems of linear function, as it is linear in positive axis. First of all, ReLu is nonlinear in nature. And combinations of ReLu are also non linear!. Great, so this means we can stack layers. It is not bound though. The range of ReLu is [0, inf]. This means it can blow up the activation. $\mathrm{ReLu}$ is less computationally expensive than tanh and sigmoid because it involves simpler mathematical operations. That is a good point to consider when we are designing deep neural nets.

Softmax classification Function: The softmax classification function is often placed at the output layer of a neural network. It's commonly used in multi-class learning problems where a set of features can be related to one-of- $\$ K \$$ classes. For example, in the CIFAR-10 word classification problem, given a set of pixels as input, we need to classify if a particular sample belongs to one-of-ten available classes. Its equation is simple, we just have to compute for the normalized exponential function of all the units in the layer. In such case,

$$
\mathrm{S}(\text { fyi })=\text { efyi } \sum \text { jefj } \mathrm{S}(\text { fyi })=\text { efyi } \sum \text { jefj }
$$

Intuitively, what the softmax does is that it squashes a vector of size KK between 00 and 11. Furthermore, because it is a normalization of the exponential, the sum of this whole vector equates to 11 . We can then interpret the output of the softmax as the probabilities that a certain set of features belongs to a certain class.

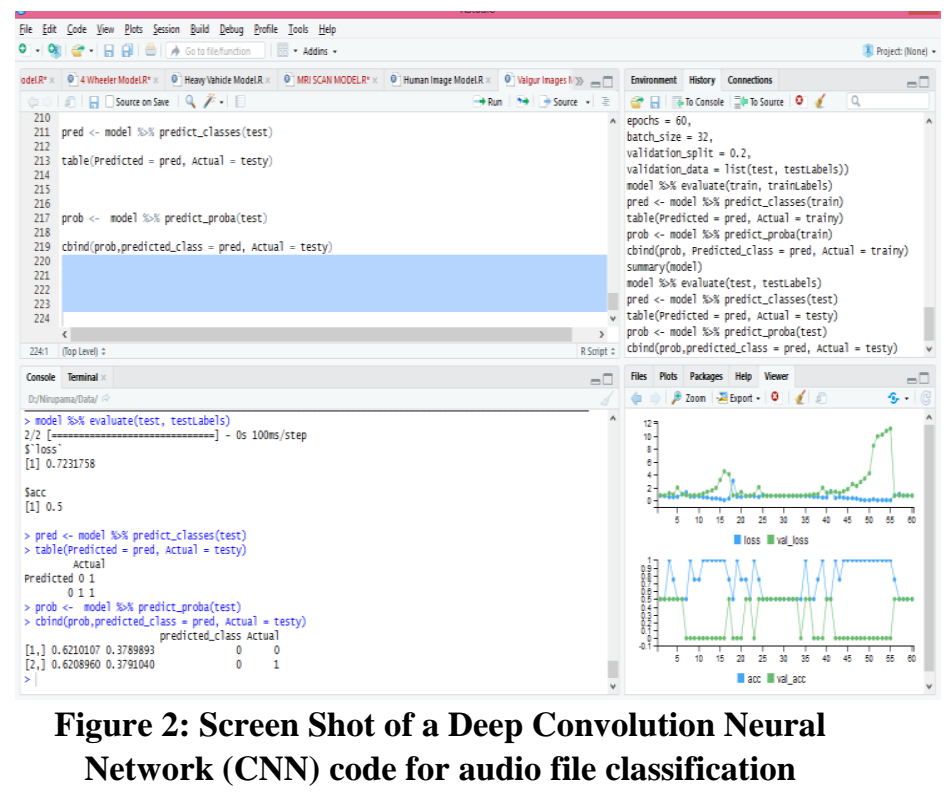

\section{CONCLUSIONS}

Social media is supposed to contribute to the maintenance of societies where democracy and multiplicity prevail could be an effective means for structuring, directing and internalizing the given ideology. Although the internet or social media are supposed to create a public sphere emancipating individuals, the fact that the social media networks are under control and surveillance refute the paper that those virtual media are really open forums. Besides, the content incorporating violence does not only produce negative effects on the users but also makes them somewhat dysfunctional.

Nevertheless, unconscious or uncontrolled use of the power of social media may lead to the spread of hate speech, infringement of personal rights, psychological attacks, symbolic violence, broadcasting private visions without the consent of the interested parties, spreading negative discourses intending to abuse, in addition to mobbing, harassment and insult along with the circulation of malevolent views and information on the Internet.

In this paper, we accomplish audio file recognition by using deep learning algorithm. We mainly apply the algorithm of deep convolution neural network to excavate the deep information of multi-layer network in the process of face recognition .And we also utilize the algorithm to make parallel computing on the cloud platform for accelerating the process of audio file recognition, analyzing theoretical acceleration ratio, and experimental verification. Experimental results show that we have achieved good results. Of course, the parallelism we do is coarse-grained, and there are still many modules that can be fine-grained in the algorithm. 


\section{REFERENCES}

[1]. Abdel-Hamid O, Mohamed A R, Jiang H, et al. Applying Convolutional Neural Networks con cepts to hybrid NN-HMM model for speech recognition[C]// Acoustics, Speech and Signal Proces sing (ICASSP), 2012 IEEE International Conference on. IEEE, 2012:4277 4280.

[2]. P.Nirupama, E. Madhusudhana Reddy, "Constrained k-Means with Dictionary Synonyms to Detect Abusing Messages in Social Network" International Journal of Pure and Applied Mathematics, ISSN: 1311-8080 (printed version), [Impact Factor: 7.19]; ISSN: 1314-3395 (on-line version), Volume 118 No. 17 2018, pp: 723-733.

[3]. P.Bhaskar, E.Madhusudhana Reddy, "Able Machine Learning Method for classifying Disease-Treatment Semantic Relations from Bio-Medical Sentences", International Journal of Recent Research Aspects (ISSN: 2349 - 7688) [Impact Factor: 2.08], Vol. 5 Issue. 1, March 2018, PP 223-226.

[4]. Kereliuk C, Sturm BL, Larsen J (2015) Deep learning and music adversaries. IEEE Trans Multimed 17(11):2059-2071.

[5]. Umberson, D., Crosnoe, R., \& Reczek, C. (2010). Social relationships and health behaviors across the life course. Annual Review of Sociology, 36, 139-157.

[6]. Umberson, D. \& Montez, J. (2010). Social relationships and health: A flashpoint for health policy. Journal of Health and Social Behavior, 51(Suppl), S54-S66.

[7]. Utz, S. \& Beukeboom , C.J. (2011). The role of social network sites in romantic relationships:effects on jealousy and relationship happiness. Journal of Computer-Mediated Communication, 16, 511-27.

[8]. Waite, L. (1995). Does marriage matter? Demography, 32, 483- 508. 